\title{
The effect of microvascular pattern alterations on network resistance in spontaneously hypertensive rats
}

\author{
Ming Yang and Walter L. Murfee \\ Department of Biomedical Engineering, Tulane University, New Orleans, LA 70118, USA
}

\section{Abstract}

Structural microvascular rarefaction, defined by a loss of vessels, is a common characteristic of hypertension and has been associated with elevated microvascular resistance. However, determining the causal relationship between microvascular network structure and resistance requires a consideration of all pattern changes throughout a network. The objectives of this study were to determine whether microvascular rarefaction is associated with other network pattern alterations and to evaluate whether pattern alterations in hypertension necessarily contribute to increased microvascular resistance. Mesenteric tissues from age-matched (15-16 weeks) male spontaneously hypertensive rats (SHR) and normotensive Wistar-Kyoto (WKY) rats were harvested and immunolabeled for PECAM. SHR networks displayed a decreased microvascular area, arteriolar-venular (AV) length, number of AV branches, and number of capillary segments. In addition, SHR networks displayed increased AV connections per network compared to WKY networks. Based on network geometries, resistance per network was calculated using a computational model. For simulations with equal vessel diameter and with relative diameters based on reported intravital measurements, SHR microvascular network resistance was not elevated compared to the WKY level. Our results suggest that microvascular pattern alterations associated with hypertension are more complex than vessel loss, and that these combined alterations do not necessarily lead to elevated resistance.

\section{Keywords}

Microcirculation; Rarefaction; Hypertension; Mesentery; Resistance; SHR

\section{Introduction}

Microvascular rarefaction, defined as an anatomical loss of microvessels, is a common characteristic of multiple tissues in hypertension. In the spontaneously hypertensive rat, rarefaction has been observed in the microvascular networks of skeletal muscle, intestine, cardiac muscle, kidney and retina $[6,26,32,37,53]$. In human patients with essential hypertension, rarefaction has also been reported in the conjunctival circulation and skin [2, $19,55]$. Because rarefaction, in some cases, precedes elevated blood pressure [23, 35], it has been assumed to play a causal role in elevated blood pressure, yet the relative contribution of microvascular network patterns associated with hypertension to microvascular resistance remains poorly understood.

Elevated microvascular resistance in hypertension has been commonly attributed to the structural abnormities at the microvascular level $[9,12,29,48]$. Intuitively, a network with 
fewer parallel flow pathways can have increased network resistance. As such, a number of studies have implicated a potential role for rarefaction in contributing to elevated network resistance, controlling blood flow and sustaining elevated blood pressure in hypertension [7, $9,18,23,27]$. Using a mathematical model of the hamster cheek pouch microcirculation, Greene et al. [18] demonstrated that a decrease in the number of vessel segments could elevate tissue resistance comparable with vessel constriction. In Greene's study, rarefaction was simulated by functionally removing vessels within normotensive networks. However, the structure alterations in networks of hypertension may be more complex than reduced number of vessels. Thus, in order to study the relative contribution of network pattern to resistance, evaluation of intact hypertensive network patterns independent of potential diameter changes is necessary.

The objectives of this study were to determine whether microvascular rarefaction is associated with other network pattern alterations and to evaluate whether pattern alterations in hypertension necessarily contribute to increased microvascular resistance. Using immunohistochemical methods, we compared the network structure of mesenteric microvascular networks from adult SHR and WKY rat strains. We then used an established network computational model $[4,43]$ to evaluate the influence of hypertensive network structure on network resistance. The rat mesentery was selected because it has been previously associated with microvascular rarefaction and dysfunction in the SHR [49, 54, 56]. We show that SHR mesenteric microvascular pattern alterations are more complex than just vessel loss, and that these alterations are not necessarily associated with increased resistance. These results challenge the existing paradigm that microvascular network structure alone is sufficient to cause increased resistance and motivate future studies aimed at identifying the functional consequences of rarefaction during hypertension.

\section{Materials and methods}

\subsection{Tissue harvesting}

All experiments were performed in accordance with the guidelines of the Tulane University Care and Use Committee. Age-matched (15-16 weeks) male SHR (6 rats) and normotensive WKY rats (4 rats) were anesthetized with IM injection of ketamine $(80 \mathrm{mg} / \mathrm{kg}$ bw, Fort Dodge Animal Health, Fort Dodge, IA, USA) and xylazine ( $8 \mathrm{mg} / \mathrm{kg}$ bw, LLOYD. Inc., Shenandoah, IA, USA), then euthanized via intra-cardiac injection of Beuthanasia (Schering-Ploug Animal Health Corp. Union, Kenilworth, NJ, USA). After making one incision through the skin and muscle layers of the abdomen, the ileum section of the mesentery was gently exteriorized. Eight mesenteric windows, identified as the thin translucent connective tissues between the artery-vein pairs feeding the small intestine, were harvested and immunolabeled.

\subsection{Immunohistochemistry}

Tissues were fixed in $4 \%$ paraformaldehyde (Fisher Scientific, Fair Lawn, NJ, USA) at $4{ }^{\circ} \mathrm{C}$ for $1 \mathrm{~h}$ or in methanol at $-20^{\circ} \mathrm{C}$ for $30 \mathrm{~min}$, then labeled with an antibody against PECAM (CD31) according to the following labeling protocol: (1) $1 \mathrm{~h}$ incubation at room temperature with 1:200 mouse monoclonal biotinylated CD31 (BD Pharmingen, San Diego, CA, USA) antibody diluted in antibody buffer (0.1\% Saponin in PBS + $2 \%$ BSA); (2) Wash with PBS with $0.1 \%$ Saponin for $30 \mathrm{~min}$; (3) $1 \mathrm{~h}$ incubation at room temperature with Streptavidinperoxidase secondary antibody solution (VECTASTAIN Elite ABC, Vector Laboratories, Burlingame, CA, USA) for $1 \mathrm{~h}$ at room temperature; and (4) $30 \mathrm{~min}$ incubation at room temperature with Vector Nova Red (Vector Laboratories) substrate. 


\subsection{Network imaging and analysis}

Images were captured using an inverted Olympus IX70 microscope (Olympus America Inc., Melville, NY, USA) coupled with a Pixel Fly camera (PCO, Kelheim, Germany) and a 4x objective (Olympus, Melville, NY, USA). Two vascularized tissues were randomly selected from each rat. The microvascular networks in these tissues were imaged. Network montages (16 WKY networks, 30 SHR networks) were generated by overlaying sequential $4 \times$ images and used for quantification of vascular area, arteriolar-venular (AV) length, the number of AV branches, capillary segments and AV connections. Vascular area was defined as the area circumscribed by all the vessels of each individual network. AV length was defined by the total length of arterioles and venules in a network. Individual arterial and venular vessels were identified based on position within the microvascular network, relative vessel diameter and endothelial cell morphology. An AV branch was defined as an arteriole or venule branching from another arteriole or venule. An AV connection was defined as a direct connection between an arteriole and a venule by a single vessel segment. All measurements were made using ImageJ software (ImageJ, U.S. National Institutes of Health, Bethesda, MO, USA).

\subsection{Network resistance calculation model}

Using a computational model, total network resistance per network was calculated across the WKY and SHR groups. Total resistance of each network was measured by dividing total pressure drop $(65 \mathrm{mmHg}$; input arteriolar pressure $=75 \mathrm{mmHg}$ and output venular pressure $=10 \mathrm{mmHg}$ ) by the total flow. Input vessels were defined as the feeding arterioles that intersect the periphery of the mesenteric tissues. Outlet vessels were defined as the draining venules that intersected the periphery of the mesenteric tissues. Feeding arteriole versus draining venule identity was determined by endothelial cell morphology and relative diameter. Endothelial cells along arterioles appeared more elongated in the direction of flow compared to cells along venules $[33,47,56]$. The $65 \mathrm{mmHg}$ pressure drop and relative input and output pressures were selected based on previous reported values used for analysis of hemodynamics within rat mesenteric microvascular networks [11, 15, 43].

Vessel segment length, the number of nodes and relative nodal position were measured for each network. Per network, vessel segments were assigned to one of five categories: feeding arterioles, pre-capillary arterioles, capillaries, post-capillary venules and draining venules. Nodes were defined as any vessel bifurcation or convergence point. These anatomical measurements were input into the model to calculate pressures and segmental flow rate. Figure 1 shows a network with identified nodes and an overview of the model algorithm.

Two diameter cases were considered for this study. For case 1, diameters for vessel segments throughout both SHR and WKY networks were set to $10 \mu \mathrm{m}$. This allowed for the delineation of microvascular pattern alteration effects from potential diameter change effects. Case 2 reflects a relative diameter scenario that is consistent with the in vivo scenario. For in vivo values, measurements from our immunohisto-chemically labeled networks were not applicable due to the potential change in vessel diameters associated with the fixation and labeling methods. This issue was especially pertinent given the evidence of increased tone in SHR vessels [44, 51]. Instead, we used the intravital measurements made by Henrich et al. [20] in adult WKY and SHR mesenteric microvascular networks, the same type of networks we used for our study. The Henrich et al. study reported the percentage differences between WKY and SHR vessels per vessel category. We applied these average increases to our networks to reflect the diameter difference in vivo between SHR networks and WKY networks. For the WKY diameters, we used the measurements from our immunohistochemically labeled WKY networks (the average diameter per vessel category: feeding arteriole $=13.9 \pm 0.9 \mu \mathrm{m}$, pre-capillary arterioles $=131.8 \pm 0.3 \mu \mathrm{m}$, capillaries $=6.2$ 
$\pm 0.1 \mu \mathrm{m}$, post-capillary venules $=16.6 \pm 0.4 \mu \mathrm{m}$ and draining venules $=18.5 \pm 1.1 \mu \mathrm{m}$ ). Then for SHR diameter values, we adjusted the WKY values by the appropriate percent increase per vessel category (percentage increases for SHR versus WKY vessels: feeding arteriole $=24.1 \%$, pre-capillary arterioles $=13.3 \%$, capillaries $=12.5 \%$, post-capillary venules $=20 \%$ and draining venules $=13.4 \%$ ).

Using the boundary conditions (i.e. input arteriolar pressure and output venular pressure), our network anatomical measurements and the diameter values per case, the nodal pressures and segmental flows were estimated assuming a modified Poiseuille Flow relationship. Use of the Poiseuille flow relationship is justified per vessel segment because of the use of apparent viscosity, which is defined based on empirical data derived from the rat mesenteric microcirculation. Individual vessel conductance, C, was computed by Eq. 1 .

$$
C=\frac{\pi D^{4}}{128 \mu \mathrm{L}}
$$

$D$ represents the diameter of the segment. $\mu$ represents the apparent viscosity of blood within the segment and $L$ is the segment length. Satisfying the conservation of mass requirement at each node within the network, the pressure at each node $(\mathrm{P})$ and segmental flow $(Q)$ can be determined from the matrix equation $\mathbf{C P}=\mathbf{Q}$ using a Matlab subroutine previously described [39]. For the first iteration, the apparent viscosity was assigned to $4 \mathrm{cP}$ and the discharge hematocrit (HD) was assigned to 0.45 . $Q$ values were used to update HD based on an empirical red blood cell distribution law (Eq. 2 [46])

$$
\begin{aligned}
& \log i t \varphi=B \log i t \frac{\psi-X_{0}}{1-2 X_{0}}+A \\
& \log i t x=\ln \frac{x}{1-x} \\
& A=-6.961\left(\frac{D_{1}}{D_{2}}\right) / D \\
& B=1+\frac{6.98\left(1-H_{D}\right.}{D} \\
& X_{0}=\frac{0.4}{D}
\end{aligned}
$$

$\psi$ is the flow fraction entering a daughter branch and $\varphi$ is the cell flux entering the same branch. $D$ is the diameter of the parent branch and $D_{1}$ and $D_{2}$ are the diameters of the daughter branches. Computed $H_{\mathrm{D}}$ values per iteration were used to update segmental viscosity $(\mu)$ per segment based on Eq. 3 [41].

$$
\begin{aligned}
& \mu=\left\{1+\left(\mu^{*}-1\right) \frac{\left(1-H_{D}\right)^{C}-1}{(1-0.45)^{C}-1}\left(\frac{D}{D-1.1}\right)^{2}\right\}\left(\frac{D}{D-1.1}\right)^{2} \\
& \mu^{*}=6 \mathrm{e}^{-0.85 D}+3.2-2.4 \mathrm{e}^{-0.06 D^{0.645}} \\
& C=\left(0.8+\mathrm{e}^{-0.075 D}\right)\left(-1+\frac{1}{1+10^{-11} D^{12}}\right) \\
& +\frac{1}{1+10^{-11} D^{12}}
\end{aligned}
$$

The segmental viscosities were then substituted back into Eq. 2 for each element in the matrix and a new network solution was obtained. Solution iterations continued until pressure in each segment changed $<0.01 \mathrm{mmHg}$ compared to the previous iteration. As mentioned above, the total network resistance was calculated by dividing the total pressure drop for the system by the total flow through the network. Thus, the resistance should largely depend on the network geometry inputs. 
Network resistance was calculated by the ratio of pressure and flow. For a Newtonian fluid, changes in resistance calculations are dependent only on geometric input changes. For our model, we allowed viscosity to change based on empirical rules that include segmental flow. To confirm that different pressure drop inputs had a negligible effect, we did calculate the resistance on nine randomly selected SHR networks with a $75 \mathrm{mmHg}$ pressure drop. The resistance difference was $0.02 \pm 0.03 \%$ different compared to the $65 \mathrm{mmHg}$ case. We also calculated resistances for the nine networks with a $95 \mathrm{mmHg}$ pressure drop. Again, resistance was minimally affected. The resistance difference was $0.35 \pm 0.1 \%$ different compared to the $65 \mathrm{mmHg}$ case.

\subsection{Statistical analysis}

Comparisons between groups were analyzed with a Mann-Whitney $U$ test. Statistical significance was accepted for $p<0.05$. Error bar values are mean \pm SEM.

\section{Results}

Consistent with the previous characterization of rarefaction in the rat mesentery [54], SHR mesenteric microvascular networks in this study were typically smaller than normotensive WKY networks (Fig. 2). Vascular area $\left(1.6 \pm 0.5 \mathrm{~mm}^{2}\right.$ vs. $\left.2.8 \pm 0.7 \mathrm{~mm}^{2}\right)$, total AV length $(6.4 \pm 1.7 \mathrm{~mm}$ vs. $12.4 \pm 2.2 \mathrm{~mm})$, number of AV braches $(4.0 \pm 0.7 \mathrm{vs} .10 .1 \pm 1.4)$ and the number of capillary segments $(10.7 \pm 1.7$ vs. $24.8 \pm 4.3)$ per network were reduced in SHR versus WKY microvascular networks (Fig. 3). This evidence indicates that SHR mesenteric networks do experience vessel loss across their hierarchy. However, microvascular network structure alterations in the SHR were more complex than just vessel loss. SHR networks displayed a significant increase in the total number of AV connections per AV length (1.99 \pm 0.27 connections $/ \mathrm{mm})$ compared to WKY networks $(0.44 \pm 0.08$ connections $/ \mathrm{mm})$. The number of larger AV connections, defined as connections with diameter larger than $10 \mu \mathrm{m}$, was also increased in SHR versus WKY networks $(0.98 \pm 0.20$ connections $/ \mathrm{mm}$ vs. $0.37 \pm$ 0.08 connections $/ \mathrm{mm}$, Fig. 4). These AV connecting vessel segments represent possible low resistance shunts from the arterial to venular side of the networks serving to reduce overall network resistance. The presence of rarefaction and the increased number of possible shunts raises the question whether SHR network structure is sufficient to increase local network resistance.

To answer this question, a network computational model was used to calculate total network resistances for the intact SHR and WKY microvascular networks. The intact pattern for the SHR networks included the characteristics of rarefaction and the increased number of AV connections. SHR and WKY resistances were compared for two diameter cases: case 1 diameters for vessel segments throughout both SHR and WKY networks were set to a uniform diameter, and case 2 diameters per vessel category adjusted to be consistent with the previously reported values measured using intravital microscopy [21]. The uniform diameter scenario allowed for isolation of the pattern contribution to total network resistance. While examples of elevated microvascular resistance were observed for SHR networks, the average SHR and WKY network resistances were not significantly different (Fig. 5a) and network resistance did not correlate with vascular area per network $(R=0.3)$. These results indicate increased resistance is not necessarily associated with rarefaction. To confirm that the uniform diameter selection did not have a significant influence for case 1 , we also compared SHR and WKY resistances setting a uniform diameter equal to $20 \mu \mathrm{m}$. No significant difference was found (data not shown). Interestingly, upon removal of AV connections from SHR networks, average SHR network resistance was significantly increased compared to the WKY average $[16.1 \pm 2.5 \mathrm{mmHg} /(\mathrm{nl} / \mathrm{min})$ vs. $8.8 \pm 1.3 \mathrm{mmHg} /$ $(\mathrm{nl} / \mathrm{min})]$. For case 2, which accounts for in vivo relative diameters, SHR network resistance was still not significantly higher compared to WKY networks (Fig. 5b). Overall, our results 
suggest that the mesenteric networks in SHRs may not necessarily have elevated resistance compared to WKY networks.

\section{Discussion}

A primary finding of this study is that microvascular network pattern alterations associated with hypertension in the SHR are more complex than vessel loss. In addition to displaying characteristics of rarefaction, SHR networks displayed an increased number of AV connections. While a reduced number of vessels contributes to elevated resistance [18], the presence of AV connections serves to offset the increased resistance by providing low resistance shunts between the arteriolar high pressure and venular low pressure sides of a network. Comparison of WKY and SHR network resistances when controlling for diameter changes (case 1: Uniform Diameter) suggest that these structural alterations alone do not necessarily result in increased microvascular resistance in the SHR mesentery. This result emphasizes the importance of evaluating resistance changes using real, intact network architectures.

The SHR represents a genetic model of hypertension and has developed elevated systemic blood pressure by 15-16 weeks compared to normotensive controls [36]. This pressure difference was confirmed for the rats used in this study by measurement of systolic arterial pressure using a tail-cuff method (SHR $189.5 \pm 9.5 \mathrm{mmHg}$ vs. WKY $123.9 \pm 12.8 \mathrm{mmHg}$ [56]). In addition to elevated blood pressure, SHR rats also display microvascular rarefaction in numerous tissues including skeletal muscle, cardiac muscle, kidney and brain of SHR $[10,18,23,25,38]$. The mesentery was selected for this study because it characteristically displays microvascular rarefaction $[34,54]$ and allows for en face visualization of entire intact network geometries. In the SHR mesentery, microvascular rarefaction has been associated with microvascular dysfunction including increased wall-tolumen ratio, enhanced microvessel specific oxidative stress, elevated MMP levels, elevated microvascular tone, deficient leukocyte-endothelial interaction and extensive non-uniform endothelial cell apoptosis $[17,24,38,44,49,50]$. Thus, this tissue offers a unique physiologically relevant model to assess the contribution of microvascular pattern alterations to increased network resistance during hypertension.

Microvascular rarefaction has been suggested as an important contributor to elevated resistance during hypertension. Rarefaction has been reported to appear during the onset of blood pressure elevation and the decreased number of vessels serves to increase resistance by providing fewer flow pathways $[1,5,9,27]$. Greene et al. demonstrated the importance of rarefaction in resistance elevation by their quantitative estimation of resistance changes after removing vessels from a network based on the normotensive hamster cheek pouch microcirculation. Our results emphasize the need to consider other network pattern alterations in addition to vessel loss when evaluating the effects of microvascular structure on resistance. This point is supported by Schulte et al.'s work [45], in which they measured forearm blood flow and pressure and reported elevated vascular resistance in hypertensive patients compared to normotensive subjects. This difference went away after maximal dilation of the vessels suggesting that network structure was not sufficient to cause the increased resistance.

Our computational approach based on intact network geometries allowed us to evaluate the influence of network pattern alterations on local microvascular resistance. Validation of the model is supported by the comparison of theoretical predictions versus experimentally measured hemodynamic parameters per vessel segment in intact Wistar mesentery networks [40, 42, 43]. Similar model algorithms have also been used for the evaluation of network hemodynamics in skeletal muscle during exercise [4]. Our results showed that SHR 
networks independent of diameter changes did not necessarily have significantly higher resistance compared WKY networks. For the networks analyzed in our study, no correlation was present between resistance and network area. We also analyzed WKY and SHR networks using relative diameter values based on intravital measurements [20]. For this scenario, SHR networks did not show different resistance compared to WKY networks. These results support that the microvascular networks associated with rarefaction do not necessarily have elevated resistance.

A limitation of our study is that our computational approach does not account for other alterations outside of segment length and relative segment position. Inputs for our model include the identification of nodal positions between the inlet and outlet vessels. This approach accounts for each vessel segment and its relative position to other upstream or downstream segments. However, the segments are assumed to be straight. Pressure losses associated with curvatures along a segment and branch angles at node positions are not directly included. Our results emphasize the importance of considering more than just vessel loss and motivate future investigation of whether these characteristics are altered during hypertensive scenarios. In addition, the networks in our study were analyzed in the absence of vascular tone, which has been reported to be elevated in the SHR [44, 51]. Hence, our flow and resistance calculations for our case 2: Relative Diameters, in which relative WKY and SHR diameters were adjusted to be consistent with the in vivo scenario, may differ from physiological values.

The quantification of the increased AV connections in adult rat mesenteric microvascular networks supports previous qualitative observations of non-tree like architectures in the SHR mesenteries [34]. The removal of these AV connections in SHR mesenteric microvascular networks resulted in an increase in resistance and a significant difference compared to WKY networks. Thus, the reported increase in AV connections provides an example of a pattern alteration that serves to offset the resistance effects contributed to rarefaction. Examples of pattern alterations also exist in other tissues. In adult SHR retinas, microvascular networks displayed increased arterio-venular crossings and loop formation [3]. In spinotrapezius muscle, SHR microvascular networks contained almost twice as many transverse arterioles with shorter branches per unit tissue volume [14]. These examples along with our results motivate the future evaluation of microvascular pattern alteration effects on resistance in other vascular beds.

The suggestion that microvascular network pattering might not influence local resistance implicates that the diameter changes may be the dominating contributor to increased peripheral resistance in hypertension. Interestingly, while vessels in SHR mesenteric networks display an increased tone [51,52], vessel segments do not have decreased diameters in vivo. Intravital observations by Henrich et al. [20] showed that the average diameter per vessel category in adult SHR mesenteric networks was higher than those of WKY networks. At the microcirculation level, other studies have also shown that small arterioles (smaller than $50 \mu \mathrm{m}$ ) in SHR do not have decreased diameter compared to normotensive control. Zweifach et al. [57] observed comparable arteriolar diameters in spinotrapezius muscle between SHR and WKY rats (5-6 and 12-13 weeks old). Similarly, small arterioles in the cremaster muscle of SHR (12-15 weeks) were reported to be as large as those in normotensive rats [30]. These studies suggest that vasoconstriction is not present in all arterioles in a microvascular bed and highlight the need to locate the exact site where vasoconstriction exists.

In order to gain insight into whether the SHR microvascular networks were associated with increased resistance when including a diameter inputs consistent with the real, in vivo scenario, we evaluated a second case using intravital measurements made in the adult 
mesenteric microvascular networks by Henrich et al. [20]. Based on the reported relative increased diameters for SHR mesenteric microvessels, resistance would not be expected to increase in our SHR versus WKY networks. Our case 2: Relative Diameters result confirms this logic and challenges the view that rarefacted SHR networks have increased resistance. Based on this result, if the flow through WKY and SHR networks is at the same level, then local pressure in the rarefacted SHR microvascular bed should not be elevated. However, increased local pressures have been reported in SHR microvascular networks from the mesentery and skeletal muscle [7, 12, 22]. An explanation for the elevated pressure measurements could be the increased resistance due to vasoconstriction of arterioles upstream from the networks which we examined. If the inlet pressure measurement location included these upstream vessel segments, then their decrease in diameter would cause increased resistance to flow and a subsequent increase in pressure. Borders and Granger [8] examined peripheral resistance in the cremaster of SHR using power dissipation theory and suggested that largest arterioles were responsible for elevated resistance. Folkow [16] suggested that sustained high blood pressure in SHR was the result of a medial hypertrophy of the walls of the larger arterioles with diameter $75-100 \mu \mathrm{m}$. Another factor could contribute to increased resistance is blood viscosity, which has been reported to be elevated in the SHR [31] and hypertensive patients [13, 28]. The relative diameters for upstream arterioles and viscosity values for SHR mesenteric microvascular networks remain unknown.

In summary, we investigated the contribution of network pattern alterations on microvascular resistance in SHR mesenteric microvascular networks. SHR networks experienced rarefaction characterized by smaller vascular area and fewer vessel segments, but did not necessarily have elevated resistance compared to WKY networks. Our results suggest that microvascular pattern alterations in the SHR are more complex than just a loss of vessels and emphasize the need to consider intact network architectures when evaluating the contribution of network structure to elevated resistance. This study offers a new perspective for understanding the influence of network structure on blood pressure elevation and motivates similar comprehensive analyses of other microvascular beds in hypertension.

\section{Acknowledgments}

This work was supported by the Tulane Hypertension and Renal Center of Excellence funded by NIH grant P20RR017659-08 (PI: L. Gabriel Navar).

\section{References}

1. Antonios TF, Singer DR, et al. Rarefaction of skin capillaries in borderline essential hypertension suggests an early structural abnormality. Hypertension. 1999; 34:655-658. [PubMed: 10523342]

2. Antonios TF, Singer DR, et al. Structural skin capillary rarefaction in essential hypertension. Hypertension. 1999; 33:998-1001. [PubMed: 10205237]

3. Bhutto IA, Amemiya T. Vascular changes in retinas of spontaneously hypertensive rats demonstrated by corrosion casts. Ophthalmic Res. 1997; 29:12-23. [PubMed: 9112262]

4. Binder KW, Murfee WL, et al. Computational network model prediction of hemodynamic alterations due to arteriolar remodeling in interval sprint trained skeletal muscle. Microcirculation. 2007; 14:181-192. [PubMed: 17454671]

5. Bobik A. The structural basis of hypertension: vascular remodeling, rarefaction and angiogenesis/ arteriogenesis. J Hypertens. 2005; 23:1473-1475. [PubMed: 16003172]

6. Bohlen HG. Intestinal microvascular adaptation during maturation of spontaneously hypertensive rats. Hypertension. 1983; 5:739-745. [PubMed: 6618636]

7. Bohlen HG, Gore RW, et al. Comparison of microvascular pressures in normal and spontaneously hypertensive rats. Microvasc Res. 1977; 13:125-130. [PubMed: 859449] 
8. Borders JL, Granger HJ. Power dissipation as a measure of peripheral resistance in vascular networks. Hypertension. 1986; 8:184-191. [PubMed: 3949373]

9. Boudier HA. Arteriolar and capillary remodelling in hypertension. Drugs. 1999; 58(Spec No 1):3740. [PubMed: 10526957]

10. Chen II, Prewitt RL, et al. Microvascular rarefaction in spontaneously hypertensive rat cremaster muscle. Am J Physiol. 1981; 241:H306-H310. [PubMed: 7282937]

11. Christensen KL, Mulvany MJ. Perindopril changes the mesenteric pressure profile of conscious hypertensive and normotensive rats. Hypertension. 1994; 23:325-328. [PubMed: 8125558]

12. DeLano FA, Schmid-Schonbein GW, et al. Penetration of the systemic blood pressure into the microvasculature of rat skeletal muscle. Microvasc Res. 1991; 41:92-110. [PubMed: 2051957]

13. Devereux RB, Case DB, et al. Possible role of increased blood viscosity in the hemodynamics of systemic hypertension. Am J Cardiol. 2000; 85:1265-1268. [PubMed: 10802017]

14. Engelson ET, Schmid-Schonbein GW, et al. The microvasculature in skeletal muscle. II. Arteriolar network anatomy in normotensive and spontaneously hypertensive rats. Microvasc Res. 1986; 31:356-374. [PubMed: 3713551]

15. Fenger-Gron J, Mulvany MJ, et al. Mesenteric blood pressure profile of conscious, freely moving rats. J Physiol. 1995; 488(Pt 3):753-760. [PubMed: 8576864]

16. Folkow B. Pathogenesis of structural vascular changes in hypertension. J Hypertens. 2004; 22:1231-1233. author reply 1234. [PubMed: 15167459]

17. Fukuda S, Yasu T, et al. Contribution of fluid shear response in leukocytes to hemodynamic resistance in the spontaneously hypertensive rat. Circ Res. 2004; 95:100-108. [PubMed: 15166092]

18. Greene AS, Tonellato PJ, et al. Microvascular rarefaction and tissue vascular resistance in hypertension. Am J Physiol. 1989; 256:H126-H131. [PubMed: 2912175]

19. Harper RN, Moore MA, et al. Arteriolar rarefaction in the conjunctiva of human essential hypertensives. Microvasc Res. 1978; 16:369-372. [PubMed: 748720]

20. Henrich H, Hertel R, et al. Structural differences in the mesentery microcirculation between normotensive and spontaneously hypertensive rats. Pflugers Arch. 1978; 375:153-159. [PubMed: 567786]

21. Henrich HA, Romen W, et al. Capillary rarefaction characteristic of the skeletal muscle of hypertensive patients. Klin Wochenschr. 1988; 66:54-60. [PubMed: 3347005]

22. Hertel R, Henrich H, Assmann R. Intravital measurement of arteriolar pressure and tangential wall stress in normotensive and spontaneously hypertensive rats. Experientia. 1978; 34(7):865-867. [PubMed: 668853]

23. Hutchins PM, Darnell AE, et al. Observation of a decreased number of small arterioles in spontaneously hypertensive rats. Circ Res. 1974; 34:161-165.

24. Inoue M. Role of oxidative stress in health and disease]. Rinsho Byori. 1996; 44:911-914. [PubMed: 8937179]

25. Kobayashi N, DeLano FA, et al. Oxidative stress promotes endothelial cell apoptosis and loss of microvessels in the spontaneously hypertensive rats. Arterioscler Thromb Vasc Biol. 2005; 25:2114-2121. [PubMed: 16037565]

26. Larouche I, Schiffrin EL. Cardiac microvasculature in DOCA-salt hypertensive rats: effect of endothelin ET(A) receptor antagonism. Hypertension. 1999; 34:795-801. [PubMed: 10523363]

27. le Noble FA, Stassen FR, et al. Angiogenesis and hypertension. J Hypertens. 1998; 16:1563-1572. [PubMed: 9856355]

28. Letcher RL, Chien S, et al. Direct relationship between blood pressure and blood viscosity in normal and hypertensive subjects. Role of fibrinogen and concentration. Am J Med. 1981; 70:1195-1202. [PubMed: 7234890]

29. Levy BI, Ambrosio G, et al. Microcirculation in hypertension: a new target for treatment? Circulation. 2001; 104:735-740. [PubMed: 11489784]

30. Lombard JH, Hess ME, et al. Neural and local control of arterioles in SHR. Hypertension. 1984; 6:530-535. [PubMed: 6746085] 
31. Lominadze D, Joshua IG, et al. Increased erythrocyte aggregation in spontaneously hypertensive rats. Am J Hypertens. 1998; 11:784-789. [PubMed: 9683038]

32. Melo RM, Martinho E Jr, et al. Training-induced, pressure-lowering effect in SHR: wide effects on circulatory profile of exercised and nonexercised muscles. Hypertension. 2003; 42:851-857. [PubMed: 12913057]

33. Murfee WL, Rehorn MR, et al. Perivascular cells along venules upregulate NG2 expression during microvascular remodeling. Microcirculation. 2006; 13:261-273. [PubMed: 16627368]

34. Murfee WL, Schmid-Schonbein GW. Chapter 12. Structure of microvascular networks in genetic hypertension. Methods Enzymol. 2008; 444:271-284. [PubMed: 19007669]

35. Noon JP, Walker BR, et al. Impaired microvascular dilatation and capillary rarefaction in young adults with a predisposition to high blood pressure. J Clin Invest. 1997; 99:1873-1879. [PubMed: 9109431]

36. Okamoto KAK. Development of a strain of spontaneously hypertensive rats. Jpn Circ J. 2009; 27:282-293. [PubMed: 13939773]

37. Paiardi S, Rodella LF, et al. Immunohistochemical evaluation of microvascular rarefaction in hypertensive humans and in spontaneously hypertensive rats. Clin Hemorheol Microcirc. 2009; 42:259-268. [PubMed: 19628891]

38. Prewitt RL, Chen II, et al. Development of microvascular rarefaction in the spontaneously hypertensive rat. Am J Physiol. 1982; 243:H243-H251. [PubMed: 7114235]

39. Price RJ, Skalak TC. A circumferential stress-growth rule predicts arcade arteriole formation in a network model. Microcirculation. 1995; 2:41-51. [PubMed: 8542539]

40. Pries AR, Reglin B, et al. Remodeling of blood vessels: responses of diameter and wall thickness to hemodynamic and metabolic stimuli. Hypertension. 2005; 46:725-731. [PubMed: 16172421]

41. Pries AR, Secomb TW, et al. Structural adaptation and stability of microvascular networks: theory and simulations. Am J Physiol. 1998; 275:H349-H360. [PubMed: 9683420]

42. Pries AR, Secomb TW, et al. Blood flow in microvascular networks. Experiments and simulation. Circ Res. 1990; 67:826-834. [PubMed: 2208609]

43. Pries AR, Secomb TW, et al. Resistance to blood flow in microvessels in vivo. Circ Res. 1994; 75:904-915. [PubMed: 7923637]

44. Schmid-Schonbein GW, Zweifach BW, et al. Microvascular tone in a skeletal muscle of spontaneously hypertensive rats. Hypertension. 1987; 9:164-171. [PubMed: 3818013]

45. Schulte KL, Braun J, et al. Functional versus structural changes of forearm vascular resistance in hypertension. Hypertension. 1988; 11:320-325. [PubMed: 3356454]

46. Secomb, TW.; Pries, AR.; Gaehtgens, P.; Gross, JF. Theoretical and experimental analysis of hematocrit distribution in microcirculatory networks. In: Lee, JS.; Skalak, TC., editors. Microvascular mechanics: hemodynamics of systemic and pulmonary microcirculation. Springer; New York: 1989. p. 39-49.

47. Stapor CP, Murfee LW. Identification of class III $\beta$-tubulin as a marker of angiogenic perivascular cells. Microvasc Res. 2012; 83(2):257-262. [PubMed: 21958528]

48. Steeghs N, Gelderblom H, et al. Hypertension and rarefaction during treatment with telatinib, a small molecule angiogenesis inhibitor. Clin Cancer Res. 2008; 14:3470-3476. [PubMed: 18519779]

49. Suematsu M, Suzuki H, et al. The inflammatory aspect of the microcirculation in hypertension: oxidative stress, leukocytes/endothelial interaction, apoptosis. Microcirculation. 2002; 9:259-276. [PubMed: 12152103]

50. Suzuki H, Schmid-Schonbein GW, et al. Impaired leukocyte-endothelial cell interaction in spontaneously hypertensive rats. Hypertension. 1994; 24:719-727. [PubMed: 7995629]

51. Suzuki H, Zweifach BW, et al. Dependence of elevated mesenteric arteriolar tone on glucocorticoids in spontaneously hypertensive rats. Int J Microcirc Clin Exp. 1995; 15:309-315. [PubMed: 8721440]

52. Suzuki H, Zweifach BW, et al. Glucocorticoid modulates vasodilator response of mesenteric arterioles in spontaneously hypertensive rats. Hypertension. 1996; 27:114-118. [PubMed: 8591872] 
53. Tomassoni D, Mancinelli G, et al. Quantitative image analysis of choroid and retinal vasculature in SHR: a model of cerebrovascular hypertensive changes? Clin Exp Hypertens. 2002; 24:741-752. [PubMed: 12450248]

54. Tran ED, Yang M, et al. Matrix metalloproteinase activity causes VEGFR-2 cleavage and microvascular rarefaction in rat mesentery. Microcirculation. 2011; 18:228-237. [PubMed: 21418372]

55. Williams SA, Boolell M, et al. Capillary hypertension and abnormal pressure dynamics in patients with essential hypertension. Clin Sci (Lond). 1990; 79:5-8. [PubMed: 2167790]

56. Yang M, Aragon M, et al. Angiogenesis in mesenteric microvascular networks from spontaneously hypertensive versus normotensive rats. Microcirculation. 2011; 18:574-582. [PubMed: 21627712]

57. Zweifach BW, Kovalcheck S, et al. Micropressure-flow relationships in a skeletal muscle of spontaneously hypertensive rats. Hypertension. 1981; 3:601-614. [PubMed: 7298115] 


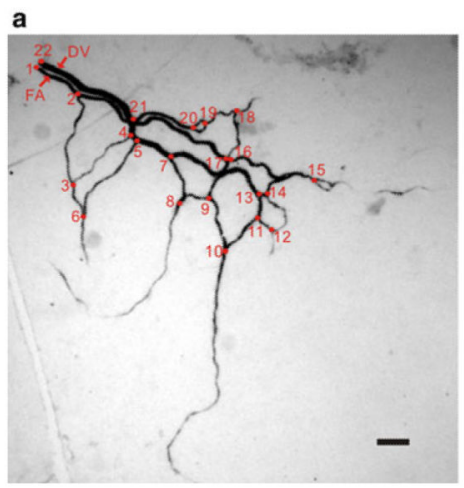

b

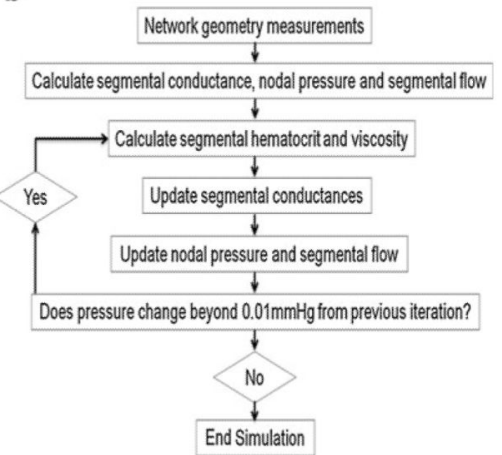

Fig. 1.

Example of SHR network and computational algorithm. a Identification of nodal positions in a PECAM labeled microvascular network. PECAM labeling identifies all microvessels within a network. Numbers indicate positions of the segment nodes. Arrows point to feeding arteriole (FA) and draining venule (DV) of the network. b Outline of the algorithm used by the network simulation. Scale bar $100 \mu \mathrm{m}$ 


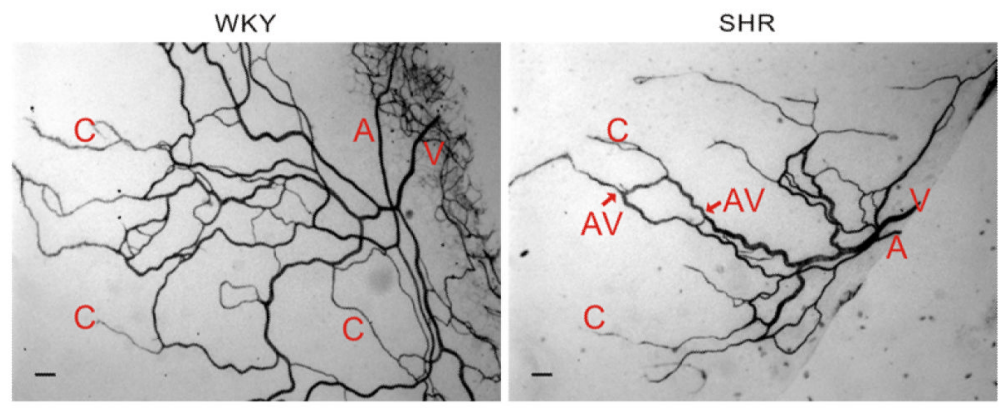

Fig. 2.

Representative images of mesenteric microvascular networks from WKY (left) and SHR (right). PECAM labeling identified the hierarchy of microvascular networks including, arterioles (A), venules (V), capillaries (C) and arteriolar-venular (AV) connections. Scale bars $100 \mu \mathrm{m}$ 

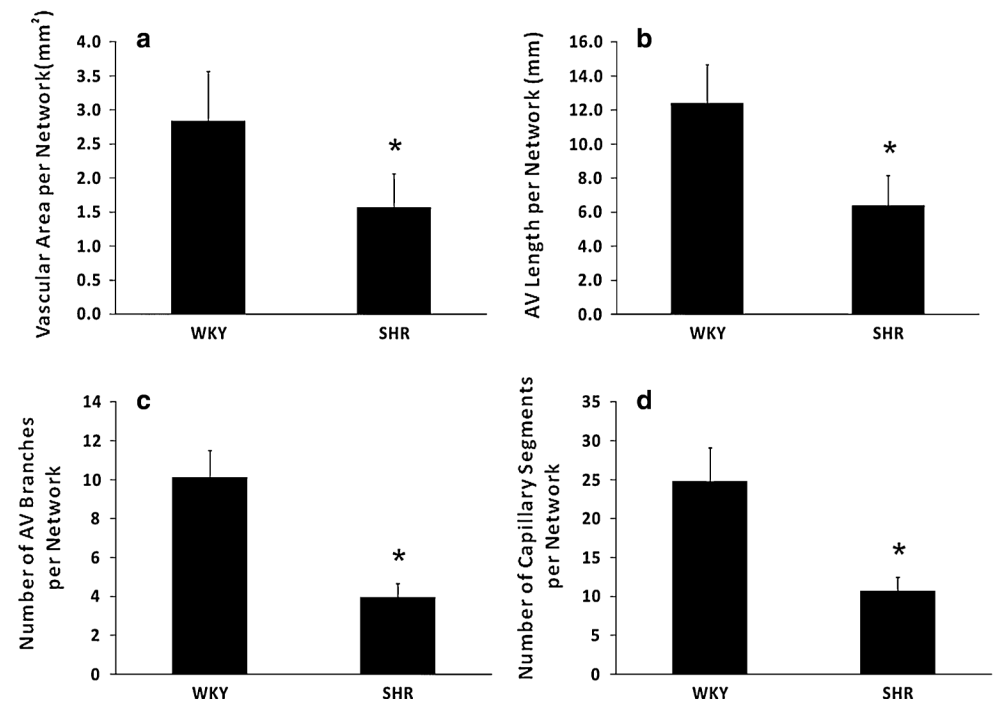

Fig. 3.

Comparisons of rarefaction metrics between SHR and WKY microvascular networks. a Vascular area per network. b Total arteriolar and venular (AV) length per network. c Number of AV branches per network. d Number of capillary segments per network. Asterisk represents significant difference between WKY and SHR $(P<0.05)$ 

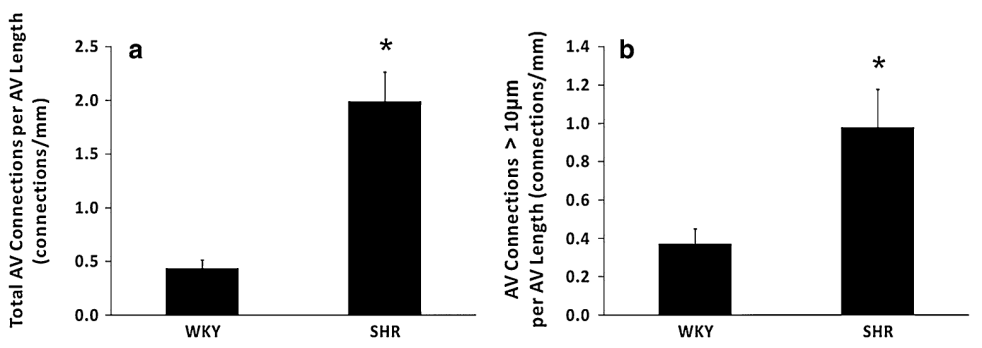

Fig. 4.

Comparisons of direct AV connections per AV length between SHR and WKY microvascular networks. a Total number of connections per AV length. b Number of connections with diameter larger than $10 \mu \mathrm{m}$ per AV length. Asterisk represents significant difference between WKY and SHR $(P<0.05)$ 

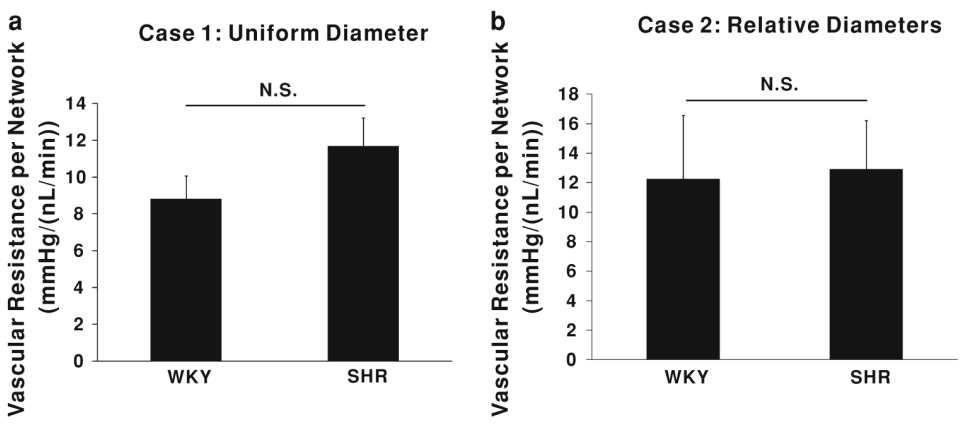

Fig. 5.

Comparisons of network resistance between SHR and WKY mesenteric networks. a For case 1 , diameters for computational simulations were set to $10 \mu \mathrm{m}$ per vessel segment for both groups. b For case 2, WKY and SHR diameters per vessel category were adjusted to be consistent with relative diameter differences previously measured using intravital microscopy [20]. N.S. represents no significant difference between WKY and SHR 